\title{
Observationally constrained modeling of sound in curved ocean internal waves: Examination of deep ducting and surface ducting at short range
}

\author{
Timothy F. Duda a) and Ying-Tsong Lin \\ Department of Applied Ocean Physics and Engineering, Woods Hole Oceanographic Institution, \\ Woods Hole, Massachusetts 02543 \\ D. Benjamin Reeder \\ Department of Oceanography, Naval Postgraduate School, Monterey, California 93943
}

(Received 19 August 2010; revised 11 April 2011; accepted 6 June 2011)

\begin{abstract}
A study of $400 \mathrm{~Hz}$ sound focusing and ducting effects in a packet of curved nonlinear internal waves in shallow water is presented. Sound propagation roughly along the crests of the waves is simulated with a three-dimensional parabolic equation computational code, and the results are compared to measured propagation along fixed 3 and $6 \mathrm{~km}$ source/receiver paths. The measurements were made on the shelf of the South China Sea northeast of Tung-Sha Island. Construction of the time-varying three-dimensional sound-speed fields used in the modeling simulations was guided by environmental data collected concurrently with the acoustic data. Computed three-dimensional propagation results compare well with field observations. The simulations allow identification of time-dependent sound forward scattering and ducting processes within the curved internal gravity waves. Strong acoustic intensity enhancement was observed during passage of high-amplitude nonlinear waves over the source/receiver paths, and is replicated in the model. The waves were typical of the region (35 $\mathrm{m}$ vertical displacement). Two types of ducting are found in the model, which occur asynchronously. One type is three-dimensional modal trapping in deep ducts within the wave crests (shallow thermocline zones). The second type is surface ducting within the wave troughs (deep thermocline zones). (C) 2011 Acoustical Society of America. [DOI: 10.1121/1.3605565]
\end{abstract}

PACS number(s): 43.30.Bp, 43.30.Re, 43.30.Dr, 43.20.El [MS] Pages: 1173-1187

\section{INTRODUCTION}

In April 2007 an acoustic propagation field study was performed on the continental shelf in the Northern South China Sea in an area where high-amplitude and steep nonlinear internal waves are common. The location was near that of the South China Sea component of the ASIAEX experiment of 2001 (Duda et al., 2004a; Chiu et al., 2004; Orr et al., 2004; Lynch et al., 2004). The April 2007 study yielded measurements of strongly time-dependent acoustic propagation properties along a pair of fixed source-to-receiver paths of 3 and $6 \mathrm{~km}$ in length, respectively, in water of about 116 $\mathrm{m}$ depth. In this paper, propagation of sound approximately along the crests of a moving curved internal-wave packet measured at the experimental site is computationally simulated. The computed time-dependent sound fields are compared against the strongly varying acoustic recordings from the field to gain insight into the processes responsible for the variability.

The oceanic measurements were summarized briefly in an earlier manuscript (Reeder et al., 2008) and in more detail in an article (Reeder et al., 2010). Internal waves and wave packets of this type are known to duct sound by trapping the energy in low-velocity ducts lying between two waves of

\footnotetext{
a) Author to whom correspondence should be addressed. Electronic mail: tduda@whoi.edu
}

depression. The waves bias depth-averaged sound speed toward greater values. Several papers have analyzed focused or ducted sound fields resulting from this deep-ducting effect for straight internal wave packets (Finette and Oba, 2003; Badiey, et al., 2005; Badiey et al., 2007; Reeder et al., 2008; Lin et al., 2009). A more recent paper has documented the acoustic effects of curved waves (Lynch et al., 2010).

Two primary acoustical effects (or processes) are reported here. First, the sound fields are found to have highly variable strong focusing, which can appear at varying time with respect to wave arrival at receiver locations, with the time offsets linked to the internal-wave curvature. Next, a surface ducting effect has been identified, causing focus structures that differ from the deep ducting effects that are already known. The computational results allow detailed study of a newly identified situation of intermingled deep ducting and surface ducting of sound within the wave packets, a consequence of which is the presence of asynchronous sweeping beams of enhanced sound energy. In addition to this highlighted result, the paper includes a description of the internal waves that were observed, including the wave characteristics that lead to the ducting. The paper also includes a description of the procedure used in the modeling. Finally, the work documents the unique fixed-path acoustic data collected at short source/receiver distances. Note that the numerical simulations allow acoustic mode coupling. The coupling effects are expected to be weak for the small angles 
between sound paths and internal-wave crests considered in this paper (Badiey et al., 2005). It is found that adiabatic mode propagation describes a majority of the findings in this work.

The experiment area is near the edge of the shelf [Fig. 1(a)]. Three types of internal wave packets exist in the

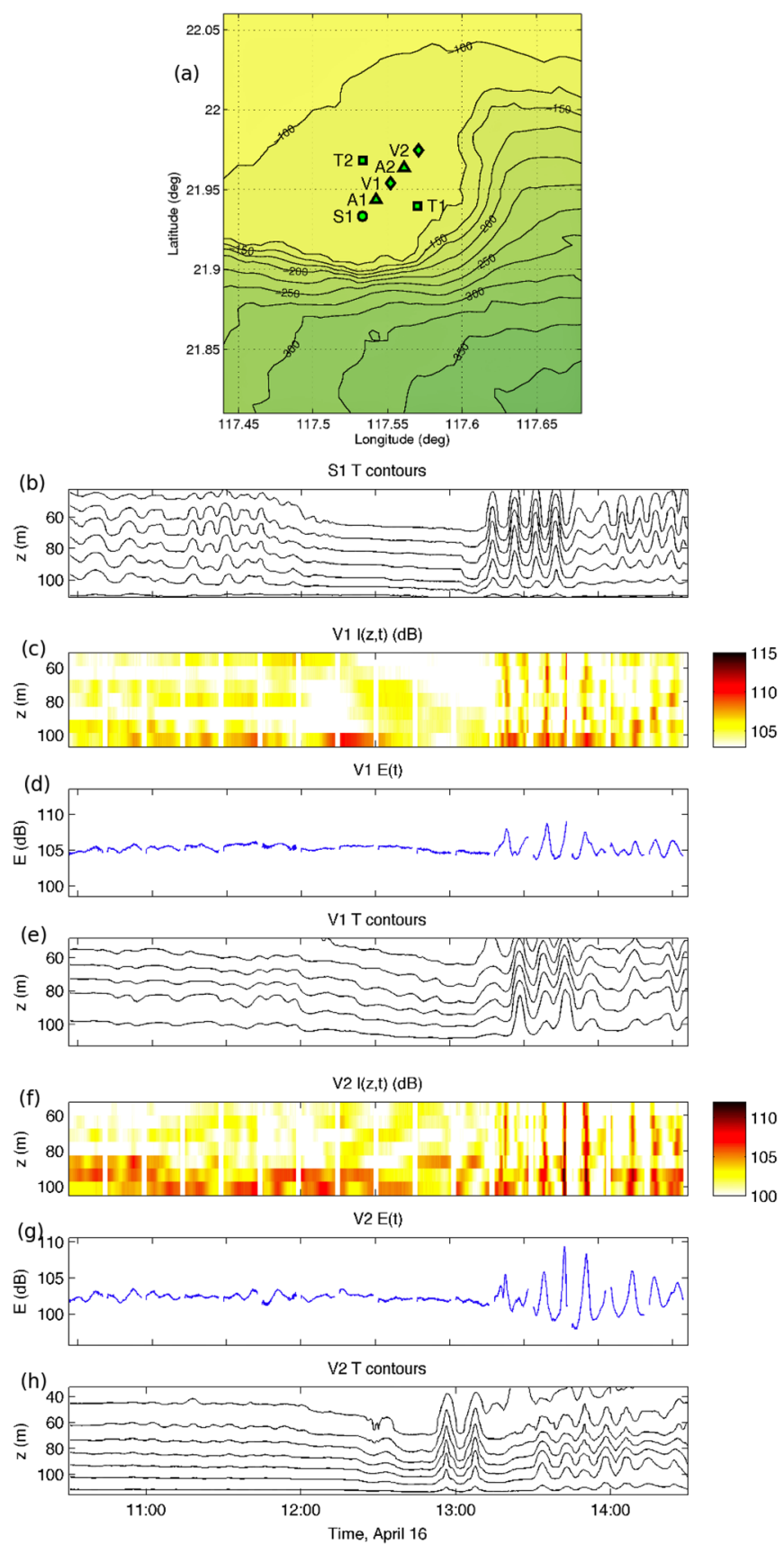

FIG. 1. (Color online) (a) A chart of the area is shown. The location is near the edge of the continental slope south of China and west of southern Taiwan. To the east and south are deep-water areas. To the west and north are shelf areas. Locations of moored instruments are shown: S1-sound source, $\mathrm{V} 1$ and V2-vertical line array receivers, A1 and A2-bottom-resting ADCPs, and T1 and T2-temperature-sensor moorings. Depth is contoured with interval of $25 \mathrm{~m}$. (b)-(h) Acoustical and environmental data are plotted together for waves of Event 1. Temperature contours show the large transbasin solitary wave arriving at S1 (b), V1 (e), and V2 (h). A train of oscillatory waves follows this wave. Intensity versus depth, $I(z, t)$ (in $\mathrm{dB}$ ) (c) and (f), and depth averaged intensity $E(t)(\mathrm{d})$ and $(\mathrm{g})$ show strong variations during the passage of the oscillatory waves. region, propagating further onto the shelf. The first type of packet consists of elevation waves formed in the tail of a deformed large transbasin nonlinear solitary wave of depression incident from deep water (Orr and Mignerey, 2003; Ramp et al., 2004). The elevation waves are possibly formed in the manner suggested by Vlasenko and Hutter (2002). This is the type whose acoustic effects are studied here. The second type of packet is formed on the shelf, created from internal tides generated in the local area (Duda et al., 2004b; Duda and Rainville, 2008). The third packet type propagates northward in the experiment area. The third type results from reflection or refraction or transbasin wave energy from Tung-Sha Island.

During the 2007 study, sound was transmitted approximately along the crests of wave packets of the first type using a moored source. The sound was received at two vertical line arrays (VLAs) at distances of 3 and $6 \mathrm{~km}$ from the source. The two sound paths were collinear. (One could also describe the experiment as having one propagation path, with measurements at two points on the path.) Large fluctuations of sound intensity (acoustic pressure squared) occurred during the experiment. These were highly correlated with the passage of packets of short and steep nonlinear internal waves [Figs. 1(b)-1(h)]. These fluctuations were expected, based on the recently published papers cited previously. The fluctuations occur because the packets correspond to sequences of ducts and antiducts for the dominant bottom-accentuated acoustic normal modes. (Most of the normal modes are bottom accentuated because of the downward refracting environment.)

Two features of the waves cause the physics of the ducting to be more complicated than the simple situation of straight parallel ducting of all normal modes. First, the waves and the associated ducts are curved in the horizontal plane. Second, deep surface ducts exist in the waves of depression. Amplitudes of the internal waves are high enough that surface convergences of well-mixed surface layer water result in very deep well-mixed layers in the waves of depression. The effect of increasing sound speed versus pressure within the nearly isothermal water thereby forms the surface ducts.

The basic physics of the deep-ducting process has been described and is not repeated in detail here (Finette and Oba, 2003; Badiey et al., 2005; Badiey et al., 2007; Lin et al., 2009). However, along-crest variability of the observed internal waves means that the results of those idealized studies with straight and uniform internal waves do not precisely apply to effects at the experiment site. Arguably, the largest deviations of the experimental situation from the idealized situations are that the internal waves are curved and change their wavelength along the $6 \mathrm{~km}$ path. This motivates threedimensional (3D) computational modeling of the sound propagation within fully $3 \mathrm{D}$ internal wave structures to attempt to understand the true effects.

The structure of the paper is as follows: Section II describes the field operations, the internal wave groups, and the acoustic intensity variations measured with VLA systems. Section III describes the three-dimensional acoustic modeling process. This includes the process used for the reconstruction of the three-dimensional ocean environment from time series data collected along a line of moorings, and 
also includes parameters of the parabolic equation propagation model. Section IV analyzes the acoustic normal modes, which will be used to interpret the observed and simulated sound fields. Section V shows the numerical modeling results. The results are interpreted and compared with experimental data. Time-dependent sound energy trapping within the surface and deep ducts is also described. Section VI is the summary.

\section{OBSERVATIONS}

The experiment took place between 12 and 22 April 2007. Figure 1(a) shows the bathymetry and the experimental configuration. The data used here are from the seven plotted moorings: S1, A1, V1, A2, V2, T1, and T2. The Taiwanese research vessel Ocean Researcher I (ORI) was used for mooring work and to collect other types of data. The first five moorings in the list were in a line at a heading of $40^{\circ} \mathrm{T}$ (relative to true north), spaced at $1.5 \mathrm{~km}$ intervals. $\mathrm{S} 1$ was the source mooring, V1 was a VLA $3 \mathrm{~km}$ distant, and V2 was a VLA $6 \mathrm{~km}$ distant. A1 and A2 were bottom-resting acoustic Doppler current profilers (ADCPs). The depth at all five of these sites was approximately $116 \mathrm{~m}$. The "S," "V," and " $\mathrm{T}$ " moorings recorded temperatures in the water column. Moorings T1 and T2 were placed such that the dominant internal waves would pass from $\mathrm{T} 1$ to the main instrument line, then to T2, providing measurements of wave speed.

The internal-wave climate in the area is known because the center of the experiment lies about $30 \mathrm{~km}$ east-northeast of the Spring 2001 ASIAEX study (Ramp et al., 2004; Duda et al., 2004a; Duda et al., 2004b). Large internal waves of depression originating west of Luzon Strait arrive here from the east. These travel at approximate heading $285^{\circ}$ (true) while they are in the deeper areas to the east of the experimental area (Ramp et al., 2004). These waves lose energy as they encounter the edge of the relatively flat shelf (Orr and Mignerey, 2003). At this point they begin to alter their form in the along-propagation direction (the across-crest direction), with the original lead depression wave stretching out greatly in length, and with simultaneous spawning of waves of elevation behind the deformed depression wave (Duda et al., 2004).

The moorings were aligned so that the path from S1 to $\mathrm{V} 1$ and thence to V2 (i.e., the two acoustic propagation paths) would be roughly parallel with the crests of passing internal waves. The waves arrive at the shelf edge with crests aligned at $5^{\circ}$ to $20^{\circ}$ true heading, based on satellite images and data from other experiments (Ramp et al., 2004). Because of the curved shape of the shelf edge and the slower wave speed on the shelf (order $0.60 \mathrm{~m} / \mathrm{s}$ ) compared to deeper water, waves were expected to be refracted into approximate alignment upon arrival at the mooring line $\left(40^{\circ} \mathrm{T}\right)$. The placement was such that the desirable situation of wave arrival at the VLAs prior to arrival at S1 might occur. This occurrence would maximize the possibility of observing sound energy deflected into the area in advance of the approaching waves (anticlockwise deflection) by the increased modal speeds in the initial depression wave. Note that this geometry did not hold for waves of "Event 1," which are examined and modeled later in this paper (i.e., Event 1 waves arrived first at S1).

The sound source was a $400 \mathrm{~Hz}$ Webb Research Corp. organ-pipe style projector at $102 \mathrm{~m}$ depth. It transmitted phase modulated m-sequence codes, repeating each $5.11 \mathrm{~s}$. Repeating sequence groups lasting $117 \mathrm{~s}$ were transmitted every 5 min from 14 April 0200 UTC (Coordinated Universal Time) to 15 April 1200 UTC. From that ending time until 20 April 0200 UTC, sequence groups lasting $13 \mathrm{~min}$ were repeated every $15 \mathrm{~min}$. Each VLA was manufactured by High Tech, Inc. and was equipped with Webb Research Corp. data recorders. Each had eight hydrophones. V1 phone depths were 51-107 m, $8 \mathrm{~m}$ separation. V2 phone depths were $52.5-105 \mathrm{~m}, 7.5 \mathrm{~m}$ in separation. Shipboard observations included acoustic backscatter visualizations of wave shapes with a SIMRAD EK500 $38 \mathrm{kHz}$ and $120 \mathrm{kHz}$ echosounder, and captured radar images of sea surface scattering.

\section{A. Internal wave groups}

The major internal-wave features at the mooring site are wave packets that form when large transbasin waves arriving from the east encounter the continental slope. After this encounter, the waves move into water of shallow depth, often of a depth less than the original maximum displacements of the waves when they were in deep water (Duda et al., 2004b; Ramp et al., 2004.). In the experimental area the waves have the form of a very wide (front to back) depression wave, which is the evolved form of a narrower transbasin depression wave (Duda et al., 2004b), followed by an oscillatory tail. Figure 2 shows five wave packet events of this type, called "Event 1" through "Event 5." Note that the shape of each wave group at V1 differs from the shape at V2 (not plotted for Event 5). This indicates that the waves have smallscale structure along their crests. This structure is evident in many satellite-derived wave images, but every structure in the images has not been confirmed to not be an artifact of surface conditions or other factors entering into the remote sensing.

Figure 2 shows that isotherms normally appearing at $60 \mathrm{~m}$ depth can descend to $80,90 \mathrm{~m}$, or more in the largest waves. Surface convergences in the leading edge of the wave are kinematically linked to depression of surface waters (and the well-mixed surface layer, if present). In the presence of a well-mixed (isothermal) layer, this can cause across-wave horizontal variations in surface duct conditions, with deeper surface ducts lying in the wave troughs. The horizontal positions of the deep surface ducts do not correspond to the deep 3D ducts, which exist between wave troughs. Figure 3 shows this effect more clearly using data from one event; mixed-layer temperatures at 32 and $65 \mathrm{~m}$ depth are equal, and are presumably close to the surface temperature (not measured), forming the surface duct, with pressure effects causing upward sound refraction. The extreme surface duct variation within internal waves is a newly recognized feature. Both sets of ducts will be shown later to have important acoustic effects (Sec. IV normal mode analysis; Sec. V simulation results).

The steep oscillatory waves in the packets have isopycnal displacement amplitudes that are quite large in comparison to 


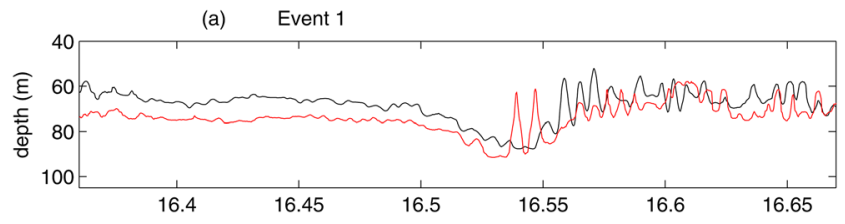

(b) Event 2
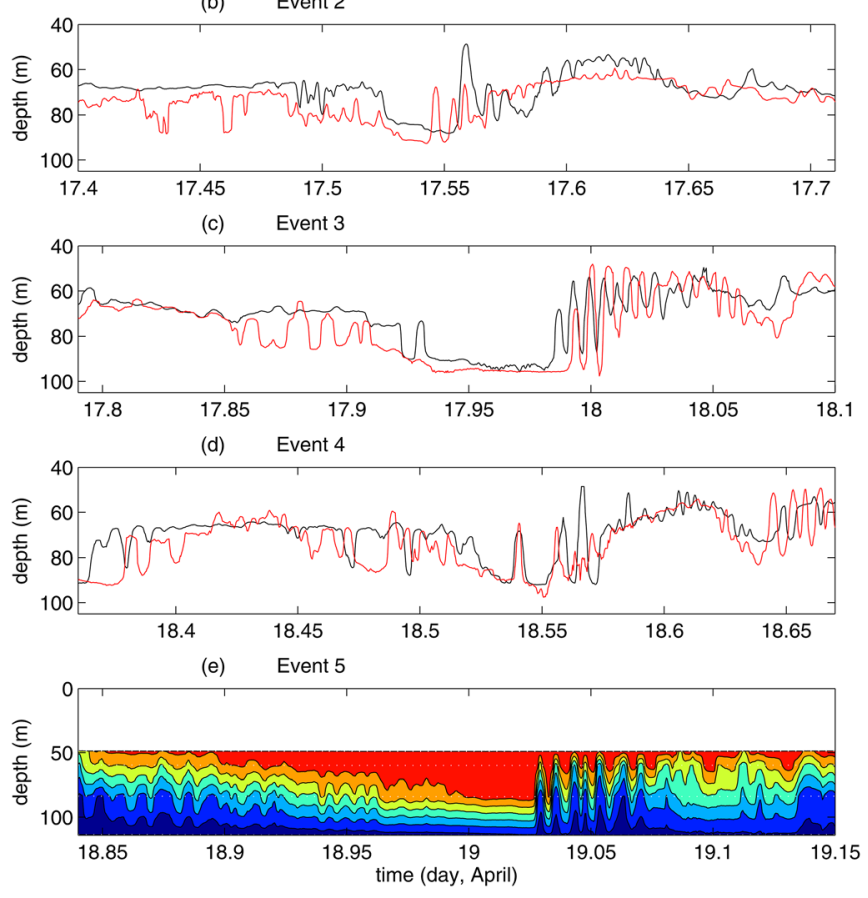

FIG. 2. (Color online) Internal waves passing the S1, V1, and V2 mooring locations are shown. Displacements of the $22{ }^{\circ} \mathrm{C}$ isotherm at $\mathrm{S} 1$ and V2 are shown starting at the top for four wave packet events: (a) mid-day April 16 (UTC), (b) mid-day April 17, (c) end-of-day April 17, and (d) mid-day April 18 (wave Events 1-4 in the terminology of this paper). Isotherm displacements are shown for V1 (black) and V2 (light tone) positions. (e) A complete set of estimated isotherm contours at V1 for another wave event, Event 5 , is shown $\left(1{ }^{\circ} \mathrm{C}\right.$ interval, $\left.20-25^{\circ} \mathrm{C}\right)$.

those seen in many areas. Comparing the observed displacements with natural length scales of the system reveals the degree of nonlinearity. In the highest waves, the ratio of the maximum displacement (from the undisturbed position outside of the wave packet) to the upper layer thickness of a two-layer system having similar mode 1 dynamics to the actual system is approximately 0.7 , seen in the lowest panel of Fig. 2. This value demonstrates appreciable nonlinearity, although much less than maximum observed nonlinearity (Vlasenko et al., 2005). The ratio of maximum displacement amplitude to water depth, an alternative wave strength indicator, is approximately 0.3 , again for the highest waves.

\section{B. Acoustic intensity variations}

Figures 1(b), 1(e), and 1(h) show strong variations of thermocline height within the wave packet of Event 1 (also shown in Fig. 2), as observed at three sites, S1, V1, and V2. The passage of the train of short waves, which follow the initial long wave, is seen to cause strong intensity variations at V1 and V2. Because the receiver arrays are very close to the source, this would be a profound focusing effect if explained by refraction within the waves. Another cause might be variable mode excitation during the passage of waves over

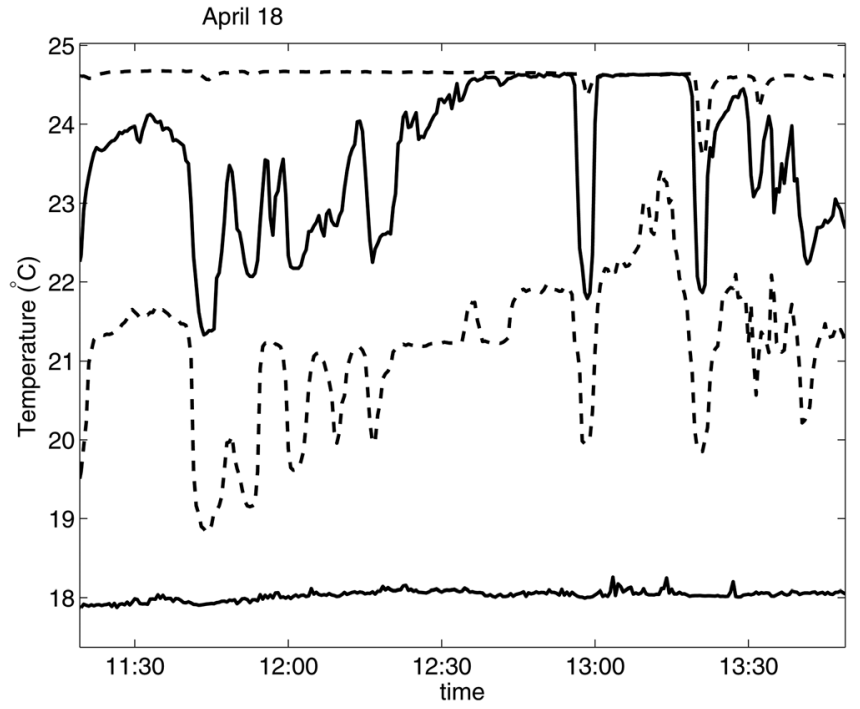

FIG. 3. Temperature records from mooring V2 on April 18 are shown. Data are from 32, 65, 87, and $114 \mathrm{~m}$ depth, warmest at $32 \mathrm{~m}$. During two depressed-thermocline type waves evident at 12:45-12:55 and 13:00-13:15 the shallowest two temperature measurements are briefly equal, indicating surface duct conditions.

the source. These two effects (refraction, altered mode excitation) are likely to occur together in nonlinear internal waves because both are ramifications of the water displacements within the waves. Long-wavelength linear internal waves may also alter mode excitation but not produce any horizontal refraction, but this paper looks primarily at effects of waves of sufficient steepness to produce significant rates of refraction. ("Sufficient steepness" is discussed in the next subsection.) Acoustic modeling can determine the relative strengths of the two effects. Altered mode excitation at the source will cause horizontally isotropic variations in intensity (absent refraction and mode coupling). Refraction (including trapping within narrow ducts) will cause strong azimuthal intensity variation (i.e., anisotropic effects). The refractive effects and sound focusing, which will be shown to be responsible for intensity fluctuations, occur because the high-amplitude internal waves contain large perturbations of sound speed from the background (at a given depth), up to $10 \mathrm{~m} / \mathrm{s}$, roughly $0.65 \%$.

\section{Internal-wave amplitude and mode refraction}

In this paper, large waves, steep waves, etc., are defined as those producing significant acoustic effects, rather than those exhibiting specific nonlinear gravity wave behavior. Thus, it is helpful at this point to define "sufficiently large" internal-wave amplitude and steepness to cause significant mode refraction in the horizontal. The variety of soundspeed profiles is such that the profile of mode phase velocity can have many forms through an internal wave, so any such criterion will be ocean model dependent (i.e. sound-speed profile dependent). In addition, the horizontal profile of wave displacement plays a role. Figure 4 is a definition sketch of the triangle-shaped waves that are used in this section. Defining significant refraction as ray deflection $\theta$ greater than $\theta_{c}$, the "sufficiency" requirement is $\cos \theta<\cos \theta_{c}$. It has been 


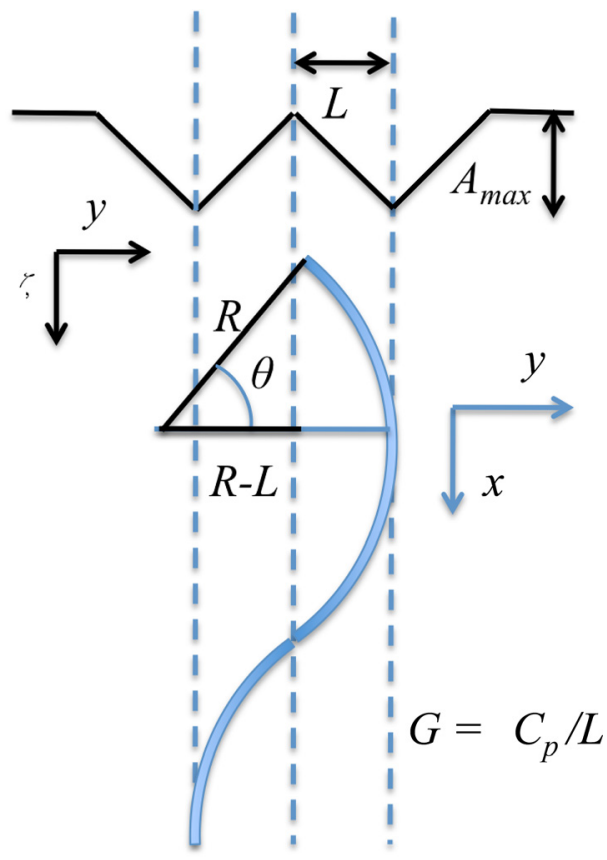

FIG. 4. (Color online) Definition sketch (view from above) for the geometry of horizontally refracted mode rays between two internal waves with triangular profiles of mode phase speed (not shown), with minimum speed in the duct center. The internal wave half-width is $L$, and the ray radius of curvature is $R$. The displacement profile is shown schematically at the top, not in plan view.

found that strong focusing and ducting in internal waves is associated with modal ray refraction of $3^{\circ}$ or greater (Lynch et al., 2010). Approximating the commonly used sech ${ }^{2}$ internal-wave profile (Apel et al., 2007) as two constant modespeed gradient linear sections (a triangle wave shape), with low mode speed between two waves of depression, yields a situation where ray segments within the duct will be circular arcs of radius of curvature $R$. Defining the half-width of the internal wave as $L$, the criterion becomes $\cos \theta=(R-L) /$ $R<\cos \theta_{c}$. Because of the relation $R=c_{p 0} / G$ for constantgradient media, where $c_{p 0}$ is a background mode phase speed and $G$ is the horizontal mode phase speed gradient in the wave (equal in magnitude for the two sides of the wave in this simple model), this is equal to $1-L G / c_{p 0}<\cos \theta_{c}$. Essentially the mode-speed anomaly amplitude, the product of the wave width $L$ and the steepness $G$ (gradient of mode speed) must be sufficiently large. Note that this product, gradient times width, is closely tied to physical wave amplitude $A$ (thermocline displacement). Examples of $L$ and $G$ can be seen in Sec. IV (normal-mode analysis).

The gradient of phase speed $G$ can be computed using perturbation forms of the modal wavenumber $k$, via $G$ $=\partial c_{p} / \partial y \approx-\omega \delta k\left(k_{0}^{2} \delta y\right)^{-1}$, where $\omega$ is the angular frequency and $k_{O}$ is a background wavenumber. The perturbation solution for the wavenumber fluctuation is $\delta k=\left(-\omega^{2} \delta c A_{\max }\right) /\left(k_{0} c_{0}^{3} H\right)$, where $\delta c$ is the difference in sound speed between the upper and lower layers, $c_{0}$ is the sound speed in the lower layer, $A_{\max }$ is the maximum internal-wave displacement, and $H$ is the depth (Lynch et al., 2010). The expressions show that $G$ is proportional to thermocline slope $\delta A / \delta y$ in this approximation, with the other terms showing the scaling of the mode-speed anomaly.
Collecting equations and approximating $c_{p 0} / c_{0}=1$ for loworder modes yields $\left(1-\cos \theta_{c}\right)<\left(\delta c / c_{0}\right) \times\left(A_{\max } / H\right)$ as a criterion for a "large wave" responsible for significant refraction. Typically, this would be solved for minimum required $A_{\text {max }}$ to give sufficient refraction. Additionally, for a wave of a given width $L$, the criterion for $A_{\max }$ yields the required wave steepness.

\section{NUMERICAL METHODS}

Simulation of 3D acoustic effects requires 3D solution of the acoustic wave equation within a volume. We use a parabolic equation marching method to find the spatial dependence of harmonic fields, beginning with a specified field at a boundary containing one sound source. Refractive index conditions within the domain are required, which is called the 3D environmental model. The model can be fully synthetic (generated algorithmically) or derived from data. Here, the South China Sea field data are used to build the water-column 3D environmental model. The procedure is described in the following subsection. The subsection after that describes the parabolic equation solution implementation.

\section{A. Three-dimensional medium interpolation process}

The 3D environmental model is constructed using data from temperature measurements at S1, V1, and V2. The $\mathrm{ADCP}$ data taken at $\mathrm{A} 1$ and $\mathrm{A} 2$ are used to verify the resultant 3D environmental model. The methods used are first briefly summarized, then explained in detail.

First, isotherm displacements are computed at the three stations. The depth profiles of displacement are fitted to the first internal-wave mode shape to determine the mode 1 amplitude as a function of location and time. With a principal component method, the two-dimensional mode amplitude $\hat{A}_{1}(x, t)$ across the fixed mooring track is generated, with wave curvature taken into account, where the $x$ axis is directed along the mooring track starting at the mooring S1. Next, the time variable is transferred into the spatial coordinate $(y)$ that is perpendicular to the $x-z$ plane using a specific internal-wave propagation speed and direction, yielding a modeled 3D field.

The procedure is now described in detail. First, a mean temperature profile is computed for each location, $T_{0}\left(x_{i}, z\right)$. The time interval for the averaging is $24 \mathrm{~h}$. For Event 1 modeling, shown later, the interval begins at 2300 on 15 April. The locations of S1, V1, and V2 are $x=x_{1}, x_{2}$, and $x_{3}$, with $y=0$. Internal-wave amplitudes are found by modeling all water column vertical displacements $(\zeta)$ as first normalmode internal-wave disturbances $\zeta\left(x_{i}, z, t\right)=A_{1}\left(x_{i}, t\right) \Phi_{1}(z)$, where $A_{1}$ is the amplitude of the first internal wave mode $\Phi_{1}$ (Apel, 1987). Writing temperature fluctuations in terms of displacements of a background temperature profile gives an expression yielding $A_{1}$,

$$
\frac{T\left(x_{i}, z, t\right)-T_{0}\left(x_{i}, z\right)}{\frac{d T_{0}\left(x_{i}, z\right)}{d z}}=A_{1}\left(x_{i}, t\right) \Phi_{1}(z) .
$$

The first mode is defined here such that positive amplitude $A_{1}$ (units of meters) corresponds to downward thermocline 
displacement. Only the first internal-wave mode is considered, consistent with many coastal internal-wave studies showing that higher modes are responsible for only small fractions of observed displacement. The shape of the mode is calculated from the background buoyancy frequency profile at the experimental site. Time-sequential least-square solution of Eq. (1) is used to estimate each of the three amplitude time series.

To interpolate $A_{1}$ between the stations, the $A_{1}\left(x_{i}, t\right)$ are time-shifted to straighten the wavefront along the $x$ axis (the mooring track), thus eliminating the effects of internal-wave curvature, using a measure of straightness that is, by necessity, somewhat arbitrary. This correction involves the arrival time delay at each station $\Delta t\left(x_{i}\right)$. The shifted first-mode amplitudes are written $A_{1}\left(x_{i}, t^{\prime}\right)$, where $t^{\prime}=t+\Delta t\left(x_{i}\right)$. Next, a principal component analysis is applied to obtain a set of empirical orthogonal functions (EOFs), $\Psi_{k}\left(t^{\prime}\right)$. These functions describe the temporal variations of $A_{1}$ at the observed three stations, with the decomposition written as

$$
A_{1}\left(x_{i}, t^{\prime}\right)=\bar{A}_{1}\left(t^{\prime}\right)+\sum_{k} \alpha_{k}\left(x_{i}\right) \Psi_{k}\left(t^{\prime}\right)
$$

with $k=1-2$. This efficiently parameterizes the time variability of the fields using only a few coefficients. For the three observed $A_{1}\left(x_{i}, t\right)$ time series, the two temporal functions form an orthogonal basis set for the three observed time series (the analysis produces a third orthonormal function that is not needed, i.e., $\alpha_{3} \approx 0$ ).

Generation of the isothermal heights between the stations is done by interpolating each EOF coefficient on the spatial grid along the $x$ axis with cubic spline fitting, yielding

$$
\hat{A}_{1}\left(x, t^{\prime}\right)=\sum_{k} \hat{\alpha}_{k}(x) \Psi_{k}\left(t^{\prime}\right),
$$

where the $\hat{\alpha}_{k}(x)$ are the interpolated coefficients of $k$ th EOF mode along the $x$ axis. For this situation of three moorings, the interpolation procedure produces $A_{1}$ that are close to simple interpolations of time-shifted $A_{1}\left(x_{i}, t^{\prime}\right)$ (i.e., EOF analysis is not required). This would not be the case for analysis of data from more than three stations using higher-order nonlinear fitting in the parameter (EOF coefficient) domain. Further, in the situation of noisy data (producing mode fitting errors), EOF set truncation would be desirable to suppress noise. Using the resultant $\hat{A}_{1}\left(x, t^{\prime}\right)$, smooth interpolation of the packet arrival time delays along the mooring track is accomplished with a cubic spline fit to the time delay measurements at the three mooring locations, defining (synthesizing) the wave curvature function $\Delta t(x)$. This function provides the smooth wave shape used in the baseline $3 \mathrm{D}$ environmental model that is studied in this paper. Small and smooth perturbation fields $\xi(x)$ can be added to the wave shape $\Delta t(x)$ to produce additional environmental models. This procedure is used later in this paper for an ad hoc error analysis. Returning the time variable to $t$ from $t^{\prime}$ over the entire track using this function produces $\hat{A}_{1}(x, t)$ and the interpolated 3D-displacement field $\eta=A \Phi$. At this point, $3 \mathrm{D}$ sound speed is computed from $\eta$ by remapping the background sound-speed profile to the displaced positions using $c_{I}(x, z, t)=c_{B}(x, z-\eta(x, z, t), t)$. A final requirement is to convert the time variable to the horizontal coordinate $y$. The best esti- mates of the speed and direction of the nonlinear waves are used, deduced by packet arrivals at T1, V1, and T2.

This environmental reconstruction procedure has been applied to wave Event 1. Figure 5(o) shows the resulting baseline mode 1 amplitude function $\hat{A}_{1}(x, t)$ along the mooring line, obtained using curvature time lags $\Delta t(x)$, i.e., $\xi(x)=0$. In Fig. 5(o), positive mode amplitude indicates downward thermocline displacement. Propagating the waves directly in the $y$ direction was found to not agree with measured wave arrival times at T1 and T2. Adjusting the wave speed vector to best agree with those data gives a direction $21^{\circ}$ anticlockwise off the $y$ axis with a speed $0.52 \mathrm{~m} / \mathrm{s}$. The wave packet position versus time is given by $[x, y]$ $=[u, v] \times\left(t-t_{0}\right)$, where $[u, v]$ is a vector of length $0.52 \mathrm{~m} / \mathrm{s}$ at heading $111^{\circ}$ anticlockwise from the mooring line (i.e., from the $x$ direction). The final model mode 1 internal-wave displacement field is plotted with the original S1, V1, and V2 data in Figs. 5(a)-5(n). Also plotted are A1 and A2 velocity data, which are compared with velocities that are consistent with the model displacements and other wave properties. The comparison shows that the model captures most of the features seen in the data. Figure 5 also shows that the waves pass T1 and T2 at the correct times. However, the detailed wave shapes differ from the model at those locations, consistent with observed nonlinear internal wave evolution in the region, not included in this model.

\section{B. Parabolic equation simulation: Setup and parameters}

The Hardin-Tappert Fourier split-step method is used to solve the parabolic wave equation in 3D Cartesian coordinates. The method is very similar to that of Lin et al. (2009). A 3D modification of the Thomson/Chapman wide-angle operator is used (Thomson and Chapman, 1983). The variable that is solved for is density-reduced pressure $p / \rho^{1 / 2}$ (acoustic pressure divided by the square root of density). The Fourier split-step method cannot solve for the impedance-reduced pressure $p(\rho c)^{-1 / 2}$ to achieve energy conservation; however, as indicated in Porter et al. (1991), using the density-reduced pressure improves the handling of density variations in the medium. Discontinuities in density, such as at the seafloor/ water interface, must be smooth in comparison to acoustic wavelength (Tappert, 1977), which prevents precise modeling of such discontinuities. The detrimental effects increase as frequency is reduced; this shortcoming does not pertain to frequencies of interest in this paper. The code is implemented in the MATLAB ${ }^{\circledR}$ environment.

The acoustic frequency $f$ of the numerical simulations is $400 \mathrm{~Hz}$. Computational parameters have been chosen through convergence testing. The range $(x)$ step is $1.0 \mathrm{~m}$, the $z$ grid spacing is $\lambda_{0} / 4$, and the $y$ grid spacing is $\lambda_{0} / 6$, where $\lambda_{0}$ is the reference acoustic wavelength with reference sound speed $c_{0}$ of $1500 \mathrm{~m} / \mathrm{s}\left(\lambda_{0}=c_{0} / f=3.75 \mathrm{~m}\right)$. In order to capture all $3 \mathrm{D}$ sound propagation features, there are $2^{10}$ vertical grid cells (including image ocean) and $2^{12} y$ direction grid cells. The water depth is $116 \mathrm{~m}$. The seafloor portion of the environmental model is spatially uniform and homogeneous (consisting of a constant sound-speed profile, 


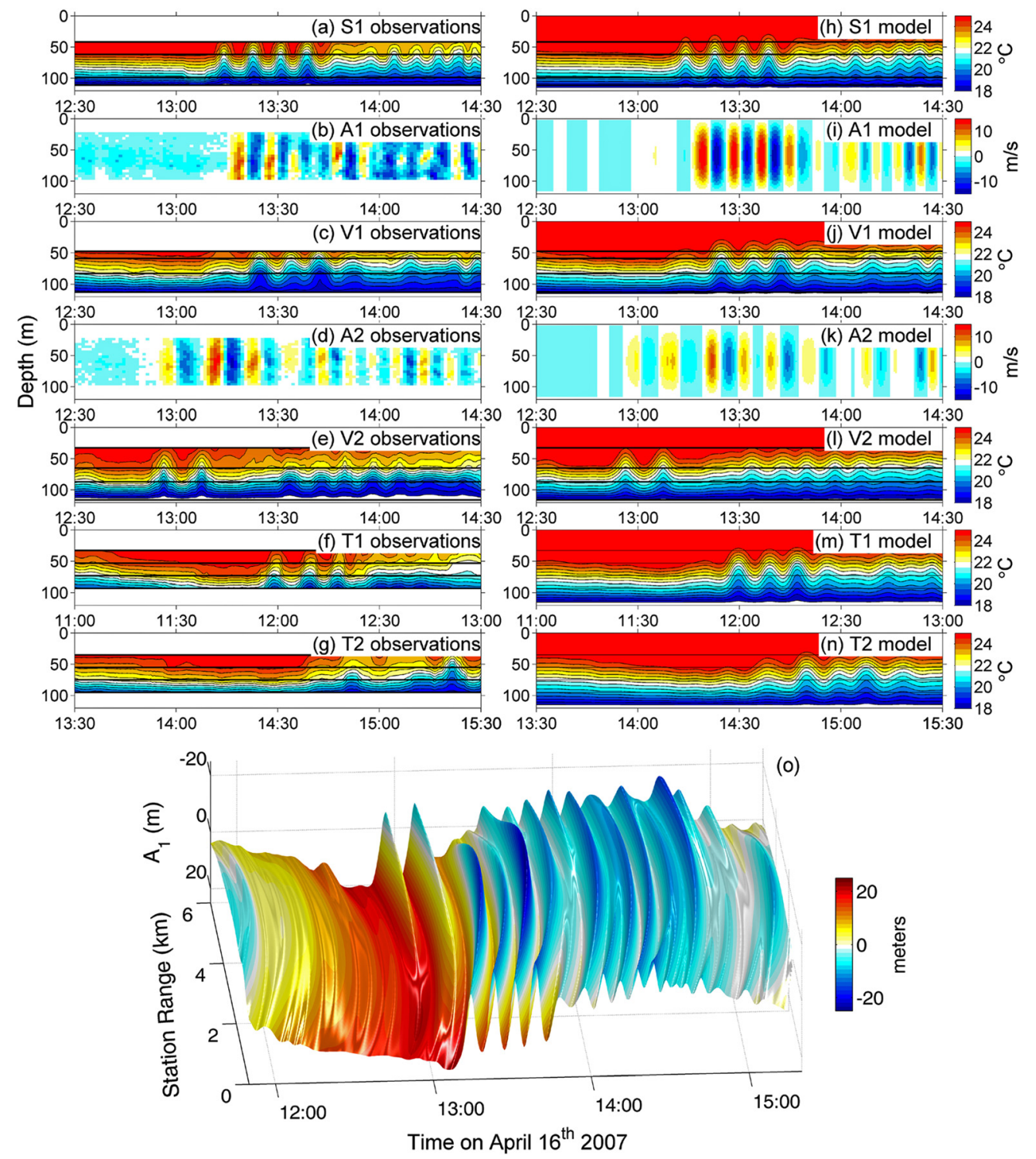

FIG. 5. (Color online) Internal wave measurements during Event 1 (mid-day April 16) at each mooring are plotted in the left panels (a)-(g). The same types of field variables (temperature or current speed) in the reconstructed internal-wave field are shown in the right panels (h)-(n). (o) The reconstructed Event-1 (mid-day April 16) mode 1 internal wave amplitude field is shown.

a constant attenuation profile, and a constant density profile). Seafloor geoacoustic parameters are: seafloor sound speed $1650 \mathrm{~m} / \mathrm{s}$; attenuation in seafloor $0.5 \mathrm{~dB}$ per wavelength; density $1500 \mathrm{~kg} / \mathrm{m}^{3}$. The seafloor parameters are derived from upper-layer measurements taken in the area (uppermost $10 \mathrm{~m}$ ) (Schock, 2004). We use the $400 \mathrm{~Hz}$ sound speed found in "medium sand" and one-half of the $400 \mathrm{~Hz}$ attenuation of "medium sand." The quoted confidence intervals on the measured attenuation are $\pm 30 \%$. Two source depths are modeled here: $102 \mathrm{~m}$, coinciding with the depth of deployed source $\mathrm{S} 1$, and $40 \mathrm{~m}$, which is a depth that will energize the surface duct. The modeled source level is $0 \mathrm{~dB}$. The source is embedded in the boundary having $x=0$. The source boundary condition is handled with the Thomson starter (Jensen et al., 1994) modified for three dimensions. The sound-speed inputs for the water column, described in the prior section, were mapped onto the computational grid using linear interpolation.

\section{ACOUSTIC NORMAL-MODE ANALYSIS}

The interpolated sound-speed field $c_{I}$ is used to study acoustic normal-mode properties and duct properties. In normal-mode theory, sound pressure in a waveguide is written

$$
p(x, y, z)=\sum_{j} C_{j}(x, y) \Theta_{j}(z ; x, y),
$$

where $C_{j}$ are the mode amplitude coefficients and $\Theta_{j}$ are the local mode vertical shapes. The mode shapes and wavenumbers $k_{j}$ are computed using the code KRAKEN (Porter, 1991) as a function of time at the mooring sites. Figure 6(b) shows example $400 \mathrm{~Hz}$ normal modes at S1 for two thermocline height scenarios: a shallow thermocline condition and a deep thermocline condition (deep surface duct). The deep condition is taken within the long initial wave of Event 1. The phase speed $c_{j}$ and attenuation $\alpha_{j}$ of mode $j$ are given by $c_{j}+i \alpha_{j}=\omega / k_{j}=\omega\left(k_{j r}+i k_{j i}\right)^{-1}=\omega\left(k_{j r}-i k_{j i}\right)|k|^{-2}$. In both 

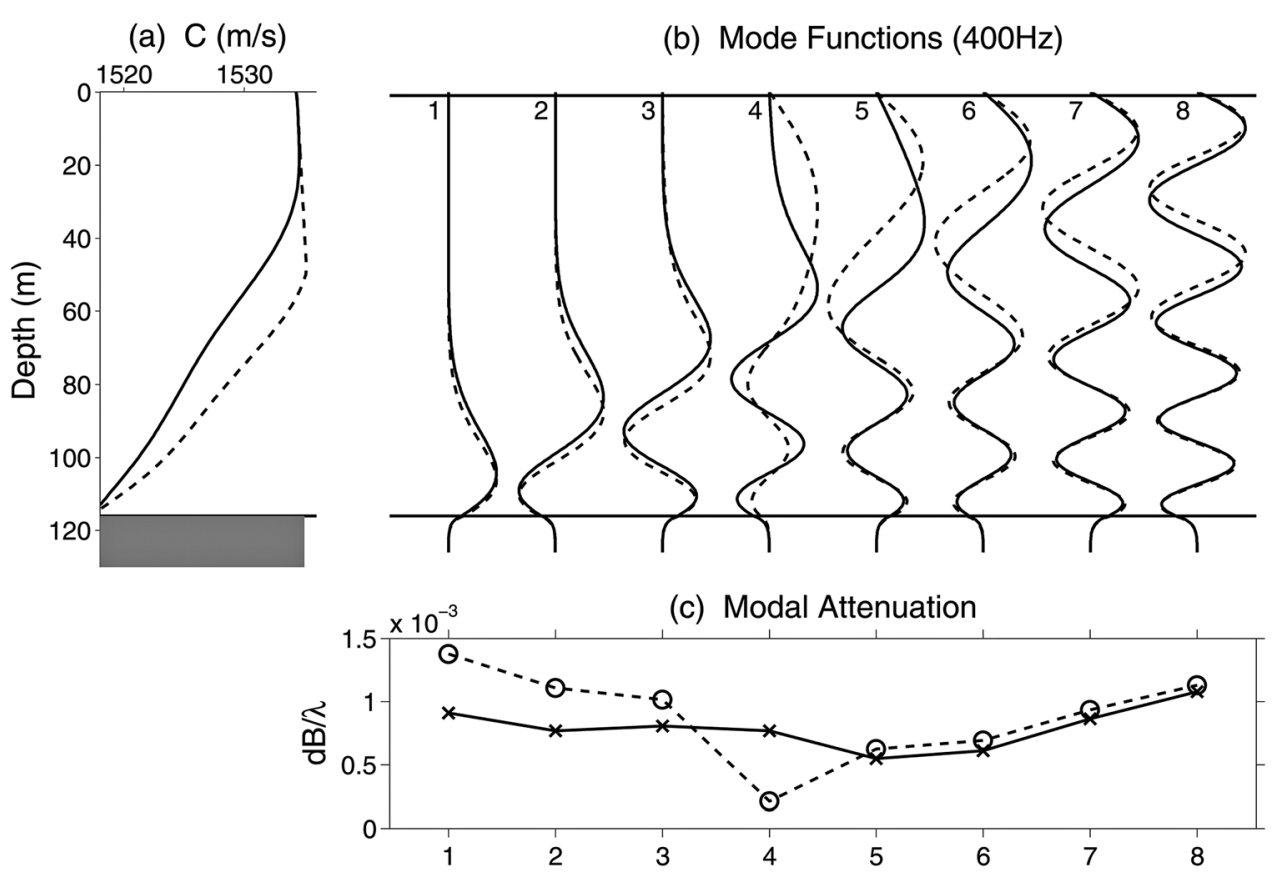

(d) Source Depth $40 \mathrm{~m}$

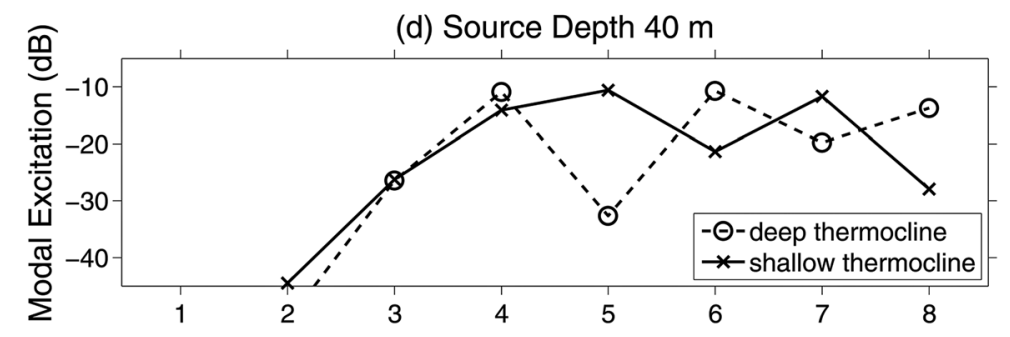

(e) Source Depth $102 \mathrm{~m}$

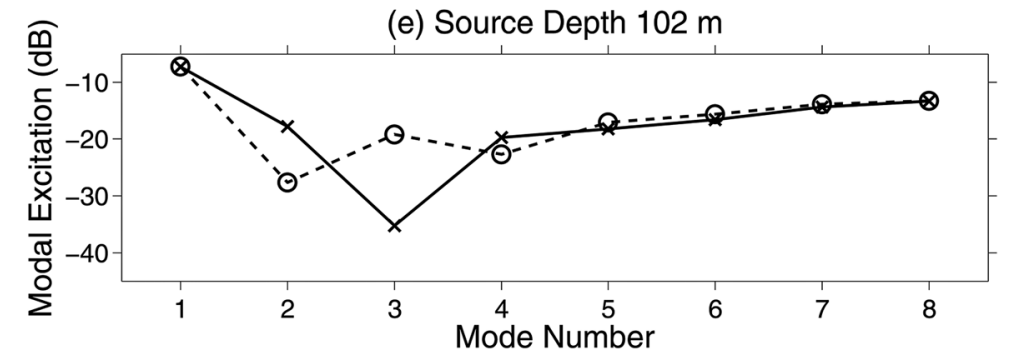

FIG. 6. (a) Water column sound speed is plotted for two conditions: Shallow thermocline (solid line) and deep thermocline (dotted-line). In all panels, solid lines indicate shallow thermocline (deep duct condition) and dotted lines indicate deep themocline (deepened surface duct condition) (b) Shapes $\Theta_{j}(z)$ of normal modes $j=1-8$ for the two conditions of (a). (c) Modal attenuation coefficients (imaginary portion of mode wavenumber $k_{j}$ ) for the two conditions. (d) Normal-mode excitations by a point $40 \mathrm{~m}$ depth source (0 dB source level) are shown for the two conditions. (e) Same as (d) for source at $102 \mathrm{~m}$ depth. situations modes 1-3 are typical bottom-trapped modes. Modes 4-6 have decreased attenuation, indicating less interaction with the seafloor. With the thermocline depressed, mode 4 is seen by its shape and low attenuation to be the mode most closely associated with surface ducting.

Figures 7(b) and 7(c) show time series of mode shapes within a portion of the Event 1 wave packet, which is shown in Fig. 7(a). What are shown are the mode shapes in the latter portion of the passing wave of depression and in the tail of shorter waves that follows. The calculation is made using the time series of displacements estimated from the S1 temperature sensors. Four example mode shapes are drawn, two each for mode 1 and mode 4 . The shape of mode 1 is altered in only a minor way by the thermocline height displacements within the waves. In contrast, mode 4 is affected strongly by the displacements. Inside the wave crests mode 4 is a typical bottom-trapped mode, whereas in large-amplitude waves of depression (wave troughs) it shifts in nature to a surface-ducted mode.
The mode phase speeds $c_{j}$ and attenuations $\alpha_{j}$ associated with the modes are plotted in Figs. 7(d) and 7(e). Modes 1 and 4 show the strongest changes in attenuation. The mode 4 attenuation perturbations are opposite in sign from the perturbations for modes $1-3$, and are consistent with alternation between surface trapped and untrapped (or weakly trapped) behaviors. Within waves of elevation in the wave packet tail, and under ambient conditions following the tail, attenuation of mode 4 increases by a factor of 4 or 5 , whereas attenuation of mode 1 decreases by about $50 \%$. The increase of mode 4 attenuation indicates the change from surface ducting to no ducting. The reduction of mode 1 attenuation results from the mode lifting away from the seabed. The depicted changes in mode phase speed indicate the relative effectiveness of the ducts (the zones of slow velocity) at horizontally trapping the various modes, with greater speed deviations indicating greater trapping proclivity. Note that all the modes can be laterally ducted in the waves of 


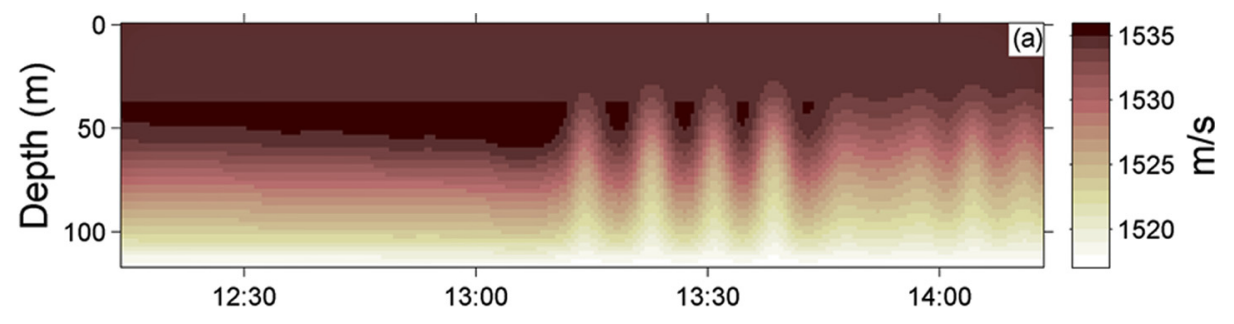

Mode 1 Function
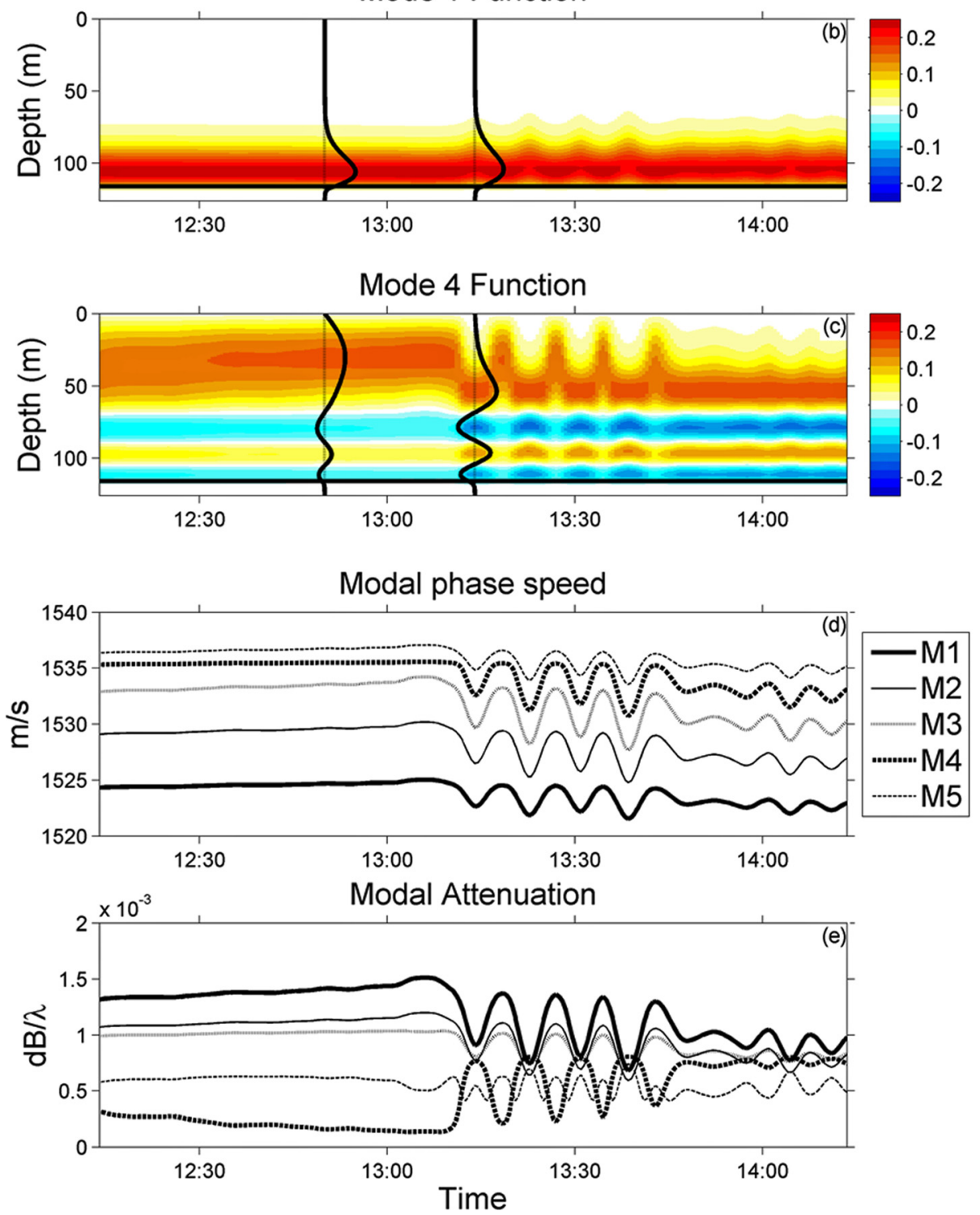

FIG. 7. (Color online) (a) Sound-speed contours at S1 in time-depth coordinates are shown for wave Event 1 of April 16. (b) The shape of acoustic normal mode one for $400 \mathrm{~Hz}$ is shown as a function of time at $\mathrm{S} 1$, computed from the interpolated field of (a). (c) Similar to (b), for mode 4. (d) Phase speeds of five acoustic normal modes $(400 \mathrm{~Hz})$ estimated from the fields in (a) are shown as a function of time. (e) Attenuation coefficients are plotted for the same five modes analyzed in (d).

elevation, and that the lateral ducting of all modes is decoupled from the vertical ducting of mode 4 (as occurring in the waves of depression). Modes 3 and 4 show the greatest reduction of phase speed in the duct, $\sim 5 \mathrm{~m} / \mathrm{s}$, with the relative index of refraction in the duct interior hovering near $1530 / 1535=0.9967$. With this value, modes 3 and 4 at grazing angles greater than about $4.6^{\circ}$ (in the duct center, with respect to the duct walls) will escape, those at lower angle will be trapped. For mode 2 this trapping (critical) angle is about $4.1^{\circ}$, maximum. For modes 1 and 5 it is about $3.6^{\circ}$ maximum.

\section{RESULTS}

The patterns that arise from the data-driven four-dimensional modeling show ducting behavior and large intensity variations, as observed in the experiment [Figs. 1(c), 1(d), $1(\mathrm{f})$, and $1(\mathrm{~g})]$. The results are analyzed in detail in this section. The basic physics of sound focusing within internalwave waveguides is straightforward. On the other hand, the patterns of beams, shadows, and interfering energy are sensitive functions of the internal-wave geometry (Lynch et al., 2010), illustrated by the modeling results. Prior to looking at 
propagation, the important effect of variable mode excitation is examined.

The resulting fields are governed strongly by modal excitation at the source. Figures 6(d) and 6(e) show $400 \mathrm{~Hz}$ modal excitation amplitudes for four situations to be analyzed in the next few sections: Source either at 102 or $40 \mathrm{~m}$ depth, and source either in an internal-wave lateral duct (elevated thermocline condition) or in an internal-wave lateral antiduct (depressed thermocline and deepened mixed layer, also the deep surface-duct condition). The $40 \mathrm{~m}$ source does not excite modes 1 and 2, and excites mode 3 weakly. The $102 \mathrm{~m}$ source excites mode 1 strongly and excites modes 2 and upward roughly equally, but less strongly than mode 1 (except for thermocline-height-dependent mode 3 excitation). Note that the $40 \mathrm{~m}$ source excites mode 4 equally for the two situations of deep or shallow thermocline; in the deep thermocline case this is a weakly attenuated surfaceduct mode [Fig. 6(c)] and this energy will propagate well, which is not the case for the shallow thermocline. Mode 5 is not excited equally by the $40 \mathrm{~m}$ source for the two thermocline positions; it is poorly excited for the deep thermocline case, and happens to be strongly excited in the shallow thermocline case (when it is the mode showing the least attenuation).
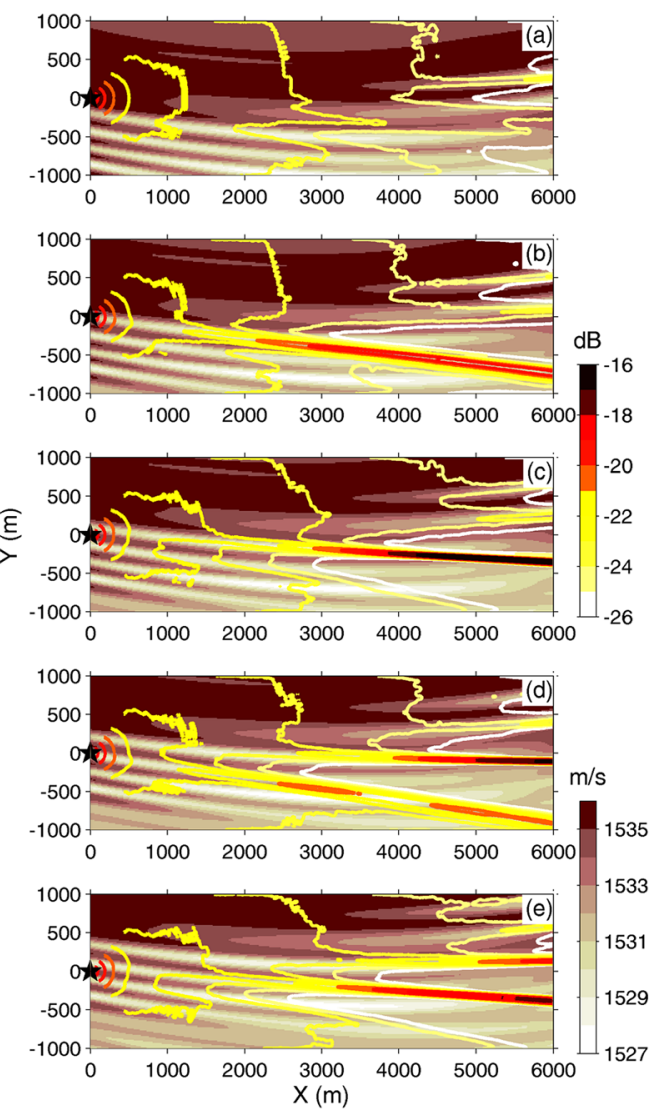

\section{A. Deep ducting}

Figure 8 shows temporal variation of $400 \mathrm{~Hz}$ sound computed for the simulated passage of the baseline $[\xi(x)=0]$ Event 1 wave packet with source depth of $102 \mathrm{~m}$ (packet depicted in Fig. 5). A time sequence of depth-averaged intensity is shown [Figs. 8(a)-8(e)]. The time sequence of a packet passing a fixed source is simulated by moving the model source position opposite the packet motion direction. The sequence shows (a) the source in the long-wavelength wave of depression (deepened surface duct situation), (b) the source in the first narrow internal-wave horizontal duct, (c) the source in the first narrow internal-wave horizontal antiduct (in the first narrow wave of depression), (d) the source in the second internal-wave duct, and (e) the source in the second antiduct.

The focused beams in the $x-y$ plane have large variations. When the source is in the wide initial wave [Fig. 8(a)], the presence of the steep (and short wavelength) nonlinear waves causes a small amount of focusing, with a maximum intensity change of about $2 \mathrm{~dB}$. Intensity perturbations of $1 \mathrm{~dB}$ can be visualized in Fig. 8(a) when an imagined circle centered at the source touches adjacent $1 \mathrm{~dB}$ contours, such as near $x=3000 \mathrm{~m}, y=-500 \mathrm{~m}$.

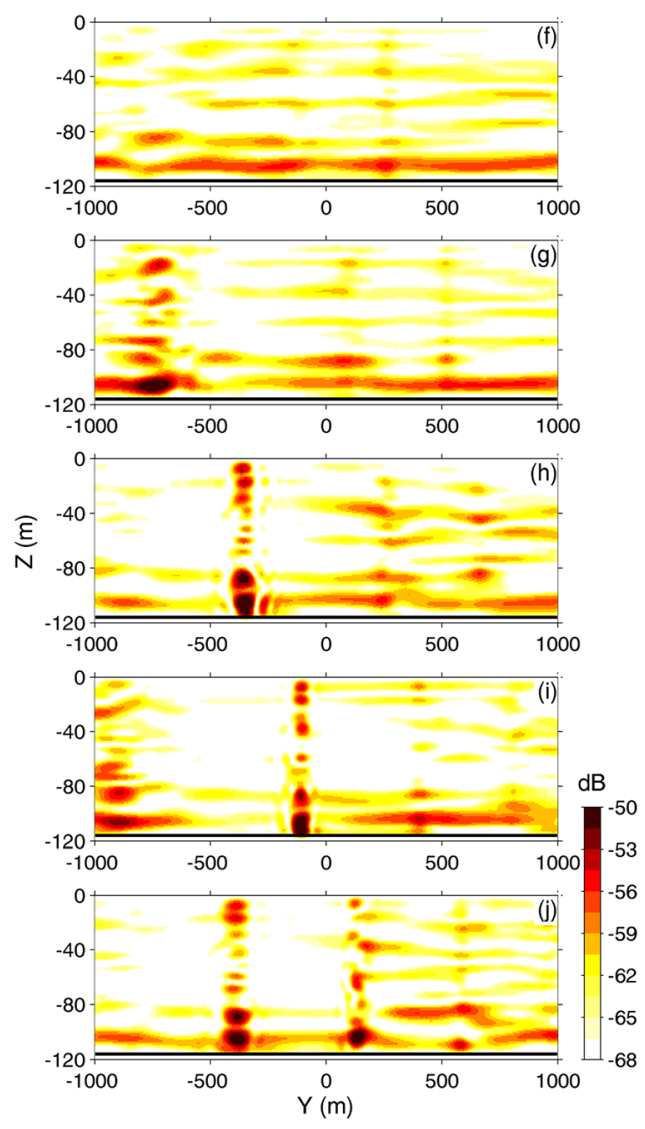

FIG. 8. (Color online) Simulated $400 \mathrm{~Hz}$ sound fields during the passage of wave Event 1 are shown [dB, with zero dB source level (unity)]. The baseline model is used. The source depth is $102 \mathrm{~m}$. On the left in panels (a)-(e) is a sequence of plan views of depth-averaged intensity for five times, shown with colored contour lines at interval $1.0 \mathrm{~dB}$ (upper scale bar), superimposed on the sound speed at $50 \mathrm{~m}$ depth shown with filled contours (lower scale bar). The star shows the source position at the origin. Additionally, intensity is multiplied by distance from the source to remove cylindrical spreading effects and to compress the dynamic range of the plots. The dark filled area at the top $(y>0)$ shows the initial wide wave of depression with high sound speed anomaly. The times for panels (a)-(e) are 1307, 1314, 1318, 1322, and 1327 UTC. On the right are the intensities (not spreading adjusted) in the vertical plane at $x=6000 \mathrm{~m}$ for the same times, top to bottom (in $\mathrm{dB}$ ), with color scale at the extreme right. 
A very intense beam forms a few minutes later when the first steep wave of elevation passes over the source (i.e., when the source is in the first deep duct) [Fig. 8(b)], with 10 $\mathrm{dB}$ or greater effect at $6 \mathrm{~km}$ range. The beam is seen to exit the curved duct about $3 \mathrm{~km}$ from the source. The beam exits because the critical angles of modes are exceeded. The packet radius of curvature of $35.8 \mathrm{~km}$ is too small to allow energy to follow the wave (the value is derived from the 250 $\mathrm{m}$ deflection of the duct as $x$ changes from 0 to $3000 \mathrm{~m}$ ). Modal rays that are critical at departure from the left-hand wall of the duct [upper as drawn in Fig. 8(b)], at $x=2000 \mathrm{~m}$, $y \sim-100 \mathrm{~m}$, will bend first clockwise then counterclockwise when moving to the right across the duct, and are supercritical when reaching the other wall of the curved duct at $x=3000 \mathrm{~m}$. Therefore, they escape the duct. Other modal rays that are subcritical along the same path may also be supercritical when encountering the other side of the duct.

It is not surprising that sound is trapped in a duct that contains the source [Fig. 8(b)]. However, Fig. 8(c) shows that the duct continues to trap sound as the source position moves from duct into antiduct. In Fig. 8(c), normal modes leaving the source parallel to the $x$ axis are subcritical at the upper wall of the duct at $x \sim 2000 \mathrm{~m}$, thus focusing in the duct. Further along in the $x$ direction the modes (sound) may escape the duct, as in Fig. 8(b), or remain ducted.

At the time when the second duct passes over the source, two ducts are strongly energized with sound [Fig. 8(d)]. The conditions just described [and depicted in Fig. $8(c)]$ that cause the first duct to be strongly energized remain in place, concurrently with conditions causing trapping in the second duct. Sound trapping in the second duct is analogous with trapping in the first duct at an earlier time [compare Figs. 8(b) and 8(d)]. In Figs. 8(d) and 8(e), a third duct is energized, lying at more positive $y$ than the two intense beams. This duct, which does not exist at the source, has a weak focus at all times.

Figures $8(\mathrm{f})-8(\mathrm{j})$ show the $6000 \mathrm{~m}$ intensity fields for the same six times. The energy can be seen to be concentrated in the lower third of the water column. The deeper peaks of intensity near 90 and $100 \mathrm{~m}$ deep in the focused beams [Figs. $8(\mathrm{~h})-8(\mathrm{j})$ ] are at least $10 \mathrm{~dB}$ above the levels otherwise found at those depths. The shallow intensity peaks in the upper $30 \mathrm{~m}$ are also approximately $10 \mathrm{~dB}$ above the levels otherwise found at those depths, but are less intense than the deep beams for this $102 \mathrm{~m}$ source depth scenario.

Figures 9(a) and 9(b) compare the four-dimensional (4D) Event 1 simulation results with the Event 1 intensity measurements. Time series of depth-averaged intensity $E(t)$ at the two VLAs at 3 and $6 \mathrm{~km}$ from the source (also shown in Fig. 1) are compared with a depth average of simulated intensity, with the average computed over the 56-110 m depth range of the VLAs. The baseline simulation results (red dashed lines) use the smoothly curved wave shape (Fig. 5) determined by $\Delta t(x)+\xi(x)$, with $\xi(x)=0$. To estimate the robustness of the baseline results, simulation results are also shown for four perturbed wave cases with non-zero $\xi(x)$. Figure 9(c) shows the wave crest shapes of the five cases. The $\xi(x)$ are low-pass filtered random series, scaled to have maximum absolute value of $50 \mathrm{~m}$, and tapered to zero at the
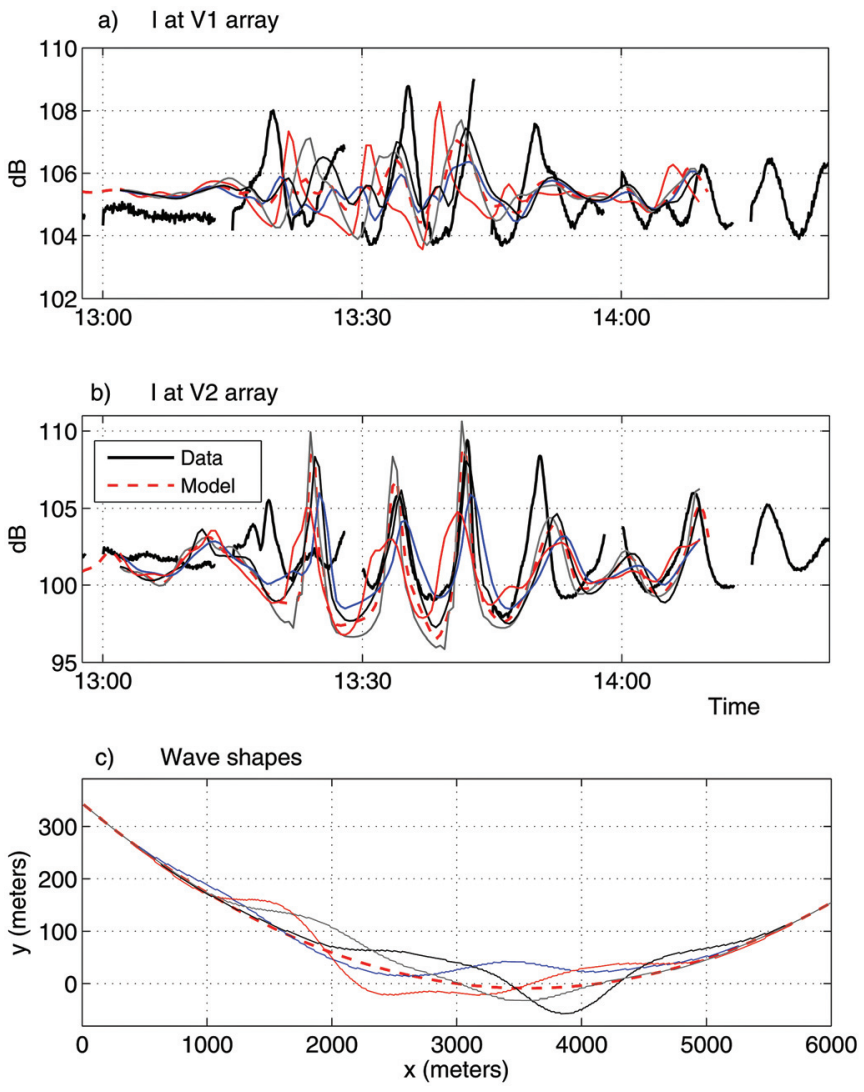

FIG. 9. Measured depth-averaged and pulse-averaged intensity $E(t)$ at V1 (frame a) and V2 (frame b) for Event 1 is shown [dark solid lines, data repeated from Figs. 2(d) and 2(g)]. Frame (c) shows the curved wave packet shape, with the baseline smooth fitted shape [from $\Delta t(x)$ ] shown with a wide red dashed line, and the perturbed shapes [from $\Delta t(x)+\xi(x)$ ] shown with thin lines. For comparison with measured $E(t)$, simulations of depth-averaged intensity time series (harmonic at $400 \mathrm{~Hz}$ ) are shown in (a) and (b) with wide red dashed lines for the baseline result (smooth wave shape). The simulation results for the four perturbed wave shapes are shown in (a) and (b) with four additional thin lines, color-coded to match (c). These models are each made by adding a perturbation curve $\xi(x)$ to the smoothly curved wave shape, with each $\xi$ curve having a maximum value near $50 \mathrm{~m}$.

ends, where the wave arrival times are well known. The $50 \mathrm{~m}$ uncertainty is equal to a wave arrival time uncertainty of less than $90 \mathrm{~s}$ (less than three temperature sample intervals). The similarities of the baseline and data intensity curves at V1 and V2 are quite strong. The five model results fit the V2 $(x=6 \mathrm{~km})$ data better than the V1 data. The five simulations give peak-trough sizes that vary by a few decibels and variable arrival time of peaks. The "red" perturbation case with $\xi(1500 \mathrm{~m})$ that is strongly positive shows the most unique results, with small intensity peaks at V2, and V1 arrival times that differ from the data and the other simulations. The other perturbation cases give results more similar to the baseline. [Note that the data-based intensity curves are averages of receptions at only eight depths, whereas the model curves are averages of 56 points within the $53 \mathrm{~m}$ aperture of the VLAs. The monochromatic model result with enhanced depth averaging (i.e., with added spatial degrees of freedom) is intended to mimic the behavior of the broadband pulse used in the field (more bandwidth degrees of freedom) averaged over fewer VLA phone depths.] 
An interesting observation from this experiment, replicated in the model, is that the intense beams of sound can be narrow, a few hundred meters across or less. In addition, the beams move quickly (roughly at the speed of the wave packets), and have short time scales for the fixed geometry of the experiment. The ranges from the source where individual beams are formed are characteristics of the beam-generation processes that are possible to examine with the model, but difficult to examine in the field. Some of the beams form 1 $\mathrm{km}$ from the source, others 3 or $5 \mathrm{~km}$ from the source. Once formed, all beams extend to the maximum simulated range of $6 \mathrm{~km}$. Intensity curves in Fig. 9 for the five simulations (four perturbation and the baseline) provide informal error bounds on the intensity amplification within the beams, and on the timing of beam arrivals. Four additional simulations were performed with waves having small perturbations $\xi(x)$. These $\xi(x)$ were those of Fig. 9(c) scaled by 0.2. These gave $E(t)$ curves falling within $0.6 \mathrm{~dB}$ of the baseline curves of Fig. 9.

\section{B. Surface ducting}

The depth excursions of the surface ducts in the packets have important effects on attenuation versus range of sound from shallow sources. With a shallower surface duct, mode 5 has the lowest attenuation (Fig. 7), so this is the mode most highly influenced by the surface duct. Mode 5 attenuation is only slightly less than the other modes, indicating weak surface ducting. With a deepened surface duct, mode 4 has the lowest attenuation (Fig. 7). In this case, the reduction of attenuation is large, and the surface ducting is much stron- ger. For both cases, attenuation increases monotonically for high-order modes beyond the minima, consistent with the notion of a mode-stripping process limiting the propagation of these modes via seafloor interaction.

Sources at specific fixed depths can pass into and out of an internal-wave perturbed surface duct that is oscillating in vertical thickness, leading to temporal variation of seafloor-induced attenuation. A shallow source that is always in the surface duct will be less affected by the passage of the packet, and a deep source will be affected the least. In the model packet, passage into and out of the surface duct occurs for a source at 20 to $45 \mathrm{~m}$ depth. To analyze this situation, the baseline model was configured with a source at $40 \mathrm{~m}$, and with all other parameters set as for the deep-source scenario shown in the previous section. This will excite only high-order modes [see Fig. 6(d)]. Figure 10 shows contours of the sound intensity averaged within the upper $50 \mathrm{~m}$ of the water column. Again, horizontal beams are formed, as with the deep source scenario (Fig. 8). Note that the fields shown in Fig. 8 (deep source) would also show beams of energy in the upper $50 \mathrm{~m}$, with the beams similar to those in Fig. 10; these are not shown. The difference between the two situations is that with the deep source these beams correspond to deeper beams, i.e., all beams are full-depth beams. The shallow-source scenario shows wide additional 0-50 m surface trapped beams away from the full-depth beams caused by the internalwave ducts. The surface-trapped beams are at $y>0$ in Figs. 10(b) and 10(c).

The surface-ducted fraction of energy is low within the first $6 \mathrm{~km}$ of the source, and may be swamped intermittently
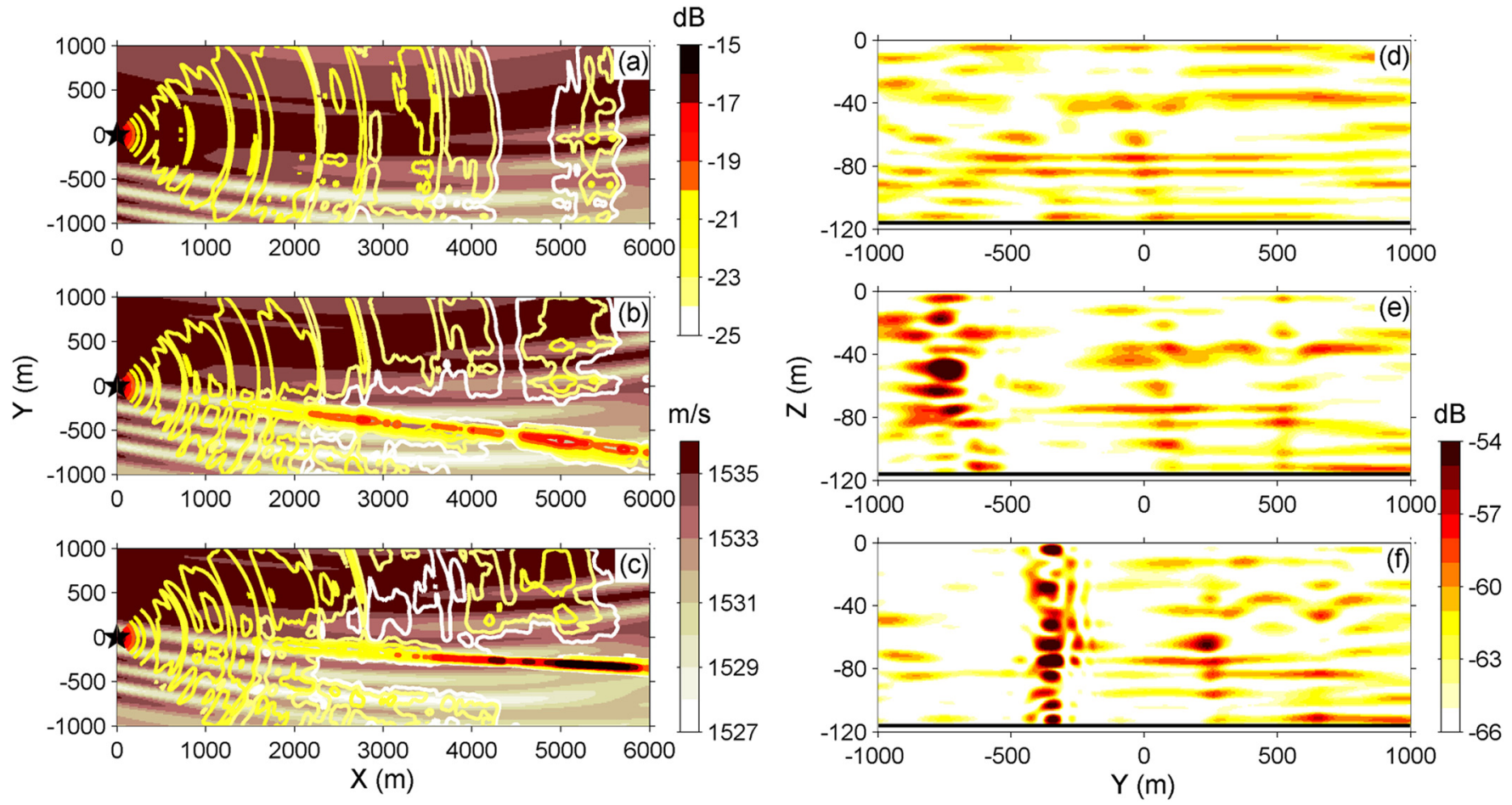

FIG. 10. (Color online) For the baseline simulation with source depth $40 \mathrm{~m}$, averages of intensity over the upper $50 \mathrm{~m}$ of the water column are contoured on the left with lines (1.0 dB interval) for three times during Event 1: (a) 1300 UTC, (b) 1314 UTC, and (c) 1318 UTC. The latter two times are the same as for Figs. 8(b) and 8(c).The filled contours show $50 \mathrm{~m}$ depth sound speed as in Figs. 8(a)-8(e). At the right, panels (d)-(f) show intensity in the plane at $x=6000 \mathrm{~m}$ for the same times. 
(a)

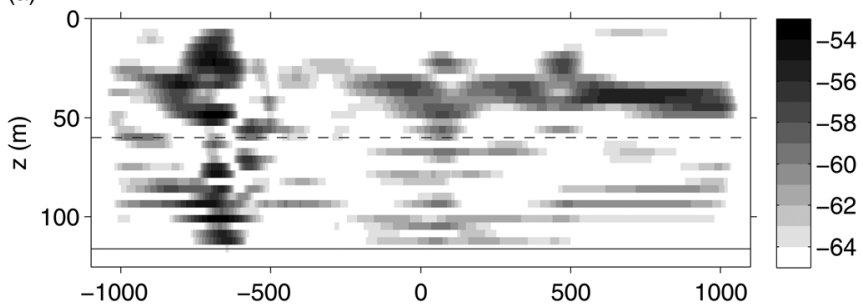

(b)

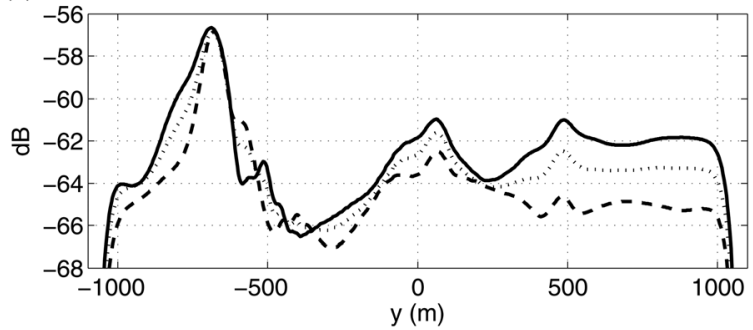

FIG. 11. (a) Baseline simulated intensity at $x=5505 \mathrm{~m}$ is plotted for Event 1, time 1314 UTC, $40 \mathrm{~m}$ source depth. (b) Average of intensity in the upper water column, above dashed line in (a) (solid line). Average of intensity in the lower water column (dashed line). Average of intensity in the entire water column (dotted line).

in range by full-depth spanning modes, but some effects can be seen nonetheless. The net effect at one range is shown in Fig. 11(a). Intensity is shown at $x=5505 \mathrm{~m}$ for Event 1, time 1314 UTC [the time of Figs. 10(b) and 10(e)]. At this time the source is below the surface duct, in a wave of elevation, which means that the source is in a horizontal duct. A beam at $y=-700 \mathrm{~m}$ is seen to trap all modes. This beam escapes from the duct at $x \sim 3000 \mathrm{~m}$. At $y$ greater than about $300 \mathrm{~m}$ the intensity in the upper half of the water column, seen in the upper right in Fig. 11(a), exceeds that in the lower half. This is consistent with strong surface ducting within the leading wide internal wave of depression. Figure 11(b) quantifies the effect more clearly. The energy in the upper half (solid line) is generally about $3 \mathrm{~dB}$ higher than the energy in the lower half for $y>300 \mathrm{~m}$. The two levels are more equal for $y<300 \mathrm{~m}$, including in the strong fulldepth beam.

A better way to evaluate surface ducting may be to look at energy in mode 4 , the weakly attenuated surface duct mode for a deep thermocline [Fig. 6(c)], and to compare it with energy in another mode that is not associated with surface ducting. In the case when the $40 \mathrm{~m}$ depth source is in the deepened mixed layer [effective surface duct situation, Figs. 10(a) and 10(d)], this mode propagates well, as expected (not shown). However, this is also the case when the $40 \mathrm{~m}$ source is not in the deepened mixed layer but is instead in the horizontal duct for bottom-trapped modes [Figs. 10(b) and 10(e)]. Figure 12(b) shows the mode 4 amplitude in this situation, found using pseudoinverse mode filtering. Figure 12(a) shows the mode 3 amplitude for comparison. Mode 4 energy is high in the full-depth beam, as expected, but it is also high outside of the beam at, e.g., $[x, y]=[6000,900]$, where this mode corresponds with surface ducted sound. The energy in this particular mode 4 beam is not associated with any deep ducts. Another interesting feature in the figure is a mode 4 beam leaving the domain at $[x, y]=[4000,-1000]$. This is consistent with mode coupling into mode 4 as the sound crosses an internal wave about $1000 \mathrm{~m}$ from the source. [Figure 10(b) shows this beam to differ from the other beams in vertical structure, and to not contain all modes.]
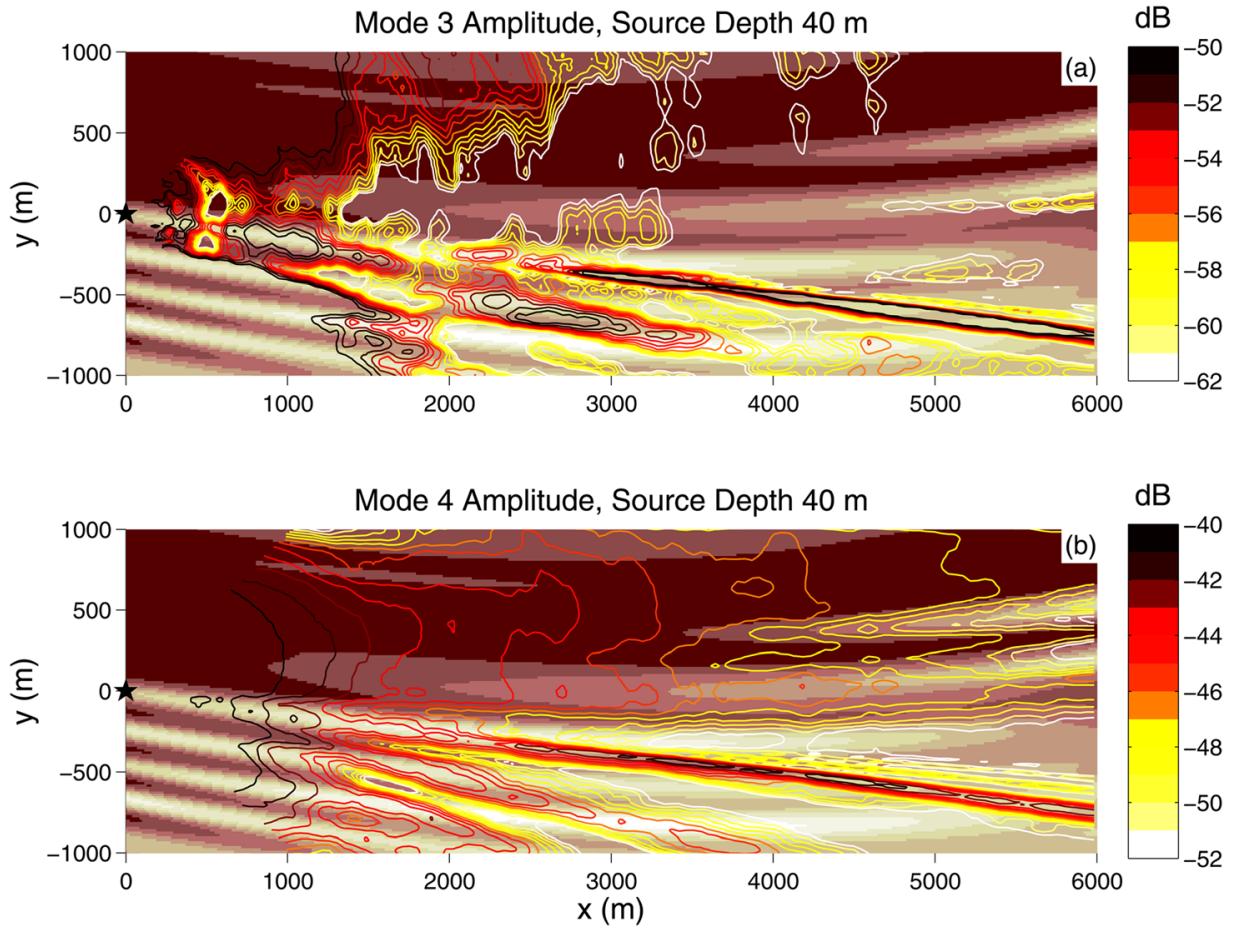

FIG. 12. (Color online) (a) Simulated mode 3 amplitude $C_{3}(x, y)$ is plotted for the source within the first internal-wave deep duct to pass by, wave Event 1, baseline wave shape [the same situation as Figs. 10(b) and 10(e)]. The shading shows $50 \mathrm{~m}$ depth sound speed as in Fig. 8, and the contours show $C_{3}(x, y)$. ( $1 \mathrm{~dB}$ contour interval.) (b) The same is shown for mode 4 , which is a surface duct mode in the area of deep thermocline (dark area). The horizontal axis ( $x$ axis) is the same for both frames. 


\section{CONCLUSIONS}

Complex patterns of sound resulting from propagation along the crests of very large and steep nonlinear internal waves have been studied using a combination of field data collected in the South China Sea and four-dimensional acoustic propagation simulations. Acoustic field data showed strong intensity fluctuations at 3 and $6 \mathrm{~km}$ from a source as internal waves passed. To help explain and understand the observations, the propagation simulations were designed to replicate conditions in the field as well as possible. To generate the internal-wave structures used in the simulations, curved internal-wave shapes were fitted to mooring temperature data collected at the source and receivers. Computed results for the situation of waves passing the site of the acoustic source show multiple time-dependent beams of intensely focused sound. These beams are created by refraction in the curved internal waves, and they agree with field observations (Fig. 9). The simulations allow the processes of sound refraction within the curved internal waves to be analyzed in detail (acoustic normal-mode refraction, in particular). Coupled-mode propagation is also very important for sound propagation through steep nonlinear internal waves. Fortunately, the simulations studied here show only a few instances of mode coupling, and the main features in the experimental data can be understood in terms of refracted adiabatic mode propagation.

The major result of the work is an explanation of the large fluctuations of sound intensity measured only a few kilometers away from the moored $400 \mathrm{~Hz}$ sound source. The strongest changes are caused by strong horizontal refraction of bottom-trapped modes away from waves of depression, toward previously recognized sound-trapping ducts (Finette and Oba, 2003). Another result that stands out is the observation that surface ducts created by large internal waves of depression create weaker secondary beams of surface-trapped energy that may not be aligned with the beams lower in the water column. The deep acoustic beams computed with the data-constrained model agree with beams measured with vertical line array receivers located in the lower half of the water column (result 1). The computed surface duct effects (result 2) could not be observed by the experimental apparatus and remain to be observed in the field.

The use of a fully 3D propagation code to model an observed situation requires a procedure for deriving the $3 \mathrm{D}$ sound-speed field. The procedure in this study uses sequential interpolation steps. Time series of internal-wave mode 1 amplitudes at each of three moorings are computed as a function of time from mooring temperature records using selected isotherm displacements. The series are then lagged to account for the curved wavefront; in other words, the wave-packet arrivals are aligned in time in some averaged sense. After interpolation using an EOF-based parameterization of the mode 1 variability the effect of the lagging is undone using cubic spline interpolation (in the $x$-direction) of the three time lags, producing a curved wave front. (For this configuration of three moorings an unlikely kinked wave front of two linear sections would also fit). Mode 1 internalwave displacements are converted to 3D sound speed using standard methods. The curved waves are marched through the domain to create a 4D wave field, without nonlinear wave evolution (an approximation and simplification) by matching the speed and direction to wave passage recorded upstream and downstream of the mooring line. Because the waves are slightly curved, there is a minor inconsistency because wave propagation direction is not everywhere normal to the wavefront.

The simulations show intermittent sound trapping and escape (Fig. 8) in the internal-wave ducts (internal waves of elevation). For a deep source in a duct, a beam will form in the duct and will then escape because of the curvature. For a deep source not in a duct, the radial sound from the source can become aligned with one or more ducts a few kilometers from the source, can focus into a beam, and may remain in the duct for a few kilometers or more. These horizontal beams of sound tend to contain many acoustic modes because the critical angles of the modes in the ducts have a small range of values.

Another process of possible importance was discovered in the course of this data-constrained experiment-motivated simulation study. The process is enhanced surface ducting (result 2), possible in the large nonlinear internal waves of depression measured in the South China Sea (Figs. 7 and 11). This effect can be analyzed in terms of the less-attenuated surface-ducted $400 \mathrm{~Hz}$ acoustic mode number 4. The effect is somewhat unanticipated, given the fact that the conditions are usually downward refracting to the extent that all $400 \mathrm{~Hz}$ acoustic modes are subject to somewhat high attenuation with the thermocline at ambient height (Figs. 6 and 7). Surface ducting is only significant in large internal waves. The large thermocline excursions in the waves will extend a mixed layer (if present) to great depth, creating laterally structured surface ducts. A unique quality of the dual-duct situation is that the sweeping full-depth horizontal beams of sound caused by the elevation-wave ducts are asynchronous with the beams of surface ducted energy that are controlled by the depression waves.

\section{ACKNOWLEDGMENTS}

Grants from the Office of Naval Research funded this work. Use of the vessels Ocean Researcher I and Ocean Researcher II in this experiment was funded by the Taiwan National Science Council. We thank Professor Chi-Fang Chen for help with the organization of this project. We are grateful to the crew members and technicians for their fine work. The science personnel aboard Ocean Researcher 1 were Dr. Barry Ma (Chief Scientist), Dr. Yung-Shen (Linus) Chiu, Wen-Hua Her, Dr. Ming-Huei Chang, Shang-Hong, Justin Chang, Dr. D. Benjamin Reeder (US Chief Scientist), Chris Miller, Marla Stone, and Keith Wykoff. Professor Ruey-Chang Wei was chief scientist of Ocean Researcher II, which also collected data associated with this study.

Apel, J. R. (1987). Principles of Ocean Physics (Academic, New York), Sec. 5.16.

Apel, J. R., Ostrovsky, L. A., Stepanyants, Y. A., and Lynch, J. F. (2007).

"Internal solitons in the ocean and their effect on underwater sound," J. Acoust. Soc. Am. 121, 695-722. 
Badiey, M., Katsnelson, B. G., Lynch, J. F., and Pereselkov, S. (2007), "Frequency dependence and intensity fluctuations due to shallow water internal waves," J. Acoust. Soc. Am. 122, 747-760.

Badiey, M., Katsnelson, B. G., Lynch, J. F., Pereselkov, S., and Siegmann, W. L. (2005). "Measurement and modeling of three-dimensional sound intensity variations due to shallow-water internal waves," J. Acoust. Soc. Am. 117, 613-625.

Chiu, C-.S., Ramp, S. R., Miller, C. W., Lynch, J. F., Duda, T. F., and Tang, T. Y. (2004). "Acoustic intensity fluctuations induced by South China Sea internal tides and solitons," IEEE J. Ocean. Eng. 29, 1249-1263.

Duda, T. F., Lynch, J. F., Irish, J. D., Beardsley, R. C., Ramp, S. R., Chiu, C.-S., Tang, T. Y., and Yang, Y. J. (2004b). "Internal tide and nonlinear internal wave behavior at the continental slope in the northern South China Sea," IEEE J. Ocean. Eng. 29, 1105-1130.

Duda, T. F., Lynch, J. F., Newhall, A. E., Wu, L., and Chiu, C.-S. (2004a). "Fluctuation of 400-Hz sound intensity in the 2001 ASIAEX South China Sea Experiment," IEEE J. Ocean. Eng. 29, 1264-1279.

Duda, T. F., and Rainville, L. (2008). "Diurnal and semidiurnal internal tide energy flux at a continental slope in the South China Sea," J. Geophys. Res. [Oceans] 113, C03025.

Finette, S., and Oba, R. (2003). "Horizontal array beamforming in an azimuthally anisotropic internal wave field," J. Acoust. Soc. Am. 114, 131-144.

Jensen, F. B., Kuperman, W. A., Porter, M. B., and Schmidt, H. (1994). Computational Ocean Acoustics (AIP, New York), Chap. 6.

Lin, Y.-T., Duda, T. F., and Lynch, J. F. (2009). "Acoustic mode radiation from the termination of a truncated nonlinear internal gravity wave duct in a shallow ocean area," J. Acoust. Soc. Am. 126, 1752-1765.

Lynch, J., Lin, Y.-T., Duda, T. F., and Newhall, A. E. (2010). "Acoustic ducting, reflection, refraction and dispersion by curved nonlinear internal waves in shallow water," IEEE J. Ocean. Eng. 35, 12-27.

Lynch, J. F., Ramp, S. R., Chiu, C.-S., Tang, T. Y., Yang, Y.-J., and Simmen, J. A. (2004). "Research highlights from the Asian Seas International Acoustics Experiment in the South China Sea," IEEE J. Ocean. Eng. 29, 1067-1074.

Orr, M. H., and Mignerey, P. C. (2003). "Nonlinear internal waves in the South China Sea: observation of the conversion of depression internal waves to elevation internal waves," J. Geophys. Res. 108, 3064.
Orr, M. H., Pasewark, B. H., Wolf, S. N., Lynch, J. F., Schroeder, T., and Chiu, C.-S. (2004). "South China Sea internal tide/internal waves-impact on the temporal variability of horizontal array gain at $276 \mathrm{~Hz}$," IEEE J. Ocean. Eng. 29, 1292-1307.

Porter, M. B., Jensen, F. B., and Ferla, C. M. (1991). "The problem of energy conservation in one-way models," J. Acoust. Soc. Am. 89, $1058-1067$.

Porter, M. B. (1991). "The KRAKEN normal mode program," SACLANTECHN Memorandum No. SM-245, SACLANT Undersea Research Center, La Spezia, Italy.

Ramp, S. R., Tang, T. Y., Duda, T. F., Lynch, J. F., Liu, A. K., Chiu, C.-S., Bahr, F., Kim, H.-R., and Yang, Y. J. (2004). "Internal solitons in the northeastern South China Sea part I: Sources and deep water propagation," IEEE J. Ocean. Eng. 29, 1157-1181.

Reeder, D. B., Chiu, L. Y. S., and Chen, C.-F. (2010). "Experimental evidence of horizontal refraction by non-linear internal waves of elevation in shallow water in the South China Sea: 3D vs. Nx2D acoustic propagation modeling," J. Comput. Acoust. 18, 267-278.

Reeder, D. B., Duda, T. F., and Ma, B. (2008). "Short-range acoustic propagation variability on a shelf area with strong nonlinear internal waves," in Oceans' 08 Kobe Conference Proceedings (IEEE, Piscataway, NJ), pp. 1-8.

Schock, S. C. (2004). "Remote estimates of physical and acoustic sediment properties in the South China Sea using chirp sonar data and the Biot model," IEEE J. Ocean. Eng. 29, 1218-1230.

Tappert, F. D. (1977). "The parabolic approximation method," in Wave Propagation and Underwater Acoustics, Lecture Notes in Physics, Vol. 70, edited by J. B. Keller and J. S. Papadakis (Springer, New York), pp. 224-287.

Thomson, D. J., and Chapman, N. R. (1983). "A wide-angle split-step algorithm for the parabolic equation," J. Acoust. Soc. Am. 74, 18481854.

Vlasenko, V., and Hutter, K. (2002). "Numerical experiments on the breaking of solitary internal waves over a slope-shelf topography," J. Phys. Oceanogr., 32, 1779-1793.

Vlasenko, V., Ostrovsky, L., and Hutter, K. (2005), "Adiabatic behavior of strongly nonlinear internal solitary waves in shelf-slope areas," J. Geophys. Res. 110, C04006. 